\title{
Analysis of genetic diversity of a native population of Myrcia lundiana Kiaersk. plants using ISSR markers
}

\author{
M.F. Alves ${ }^{1}$, D.A.C. Nizio ${ }^{1}$, F.A. Brito ${ }^{1}$, T.S. Sampaio ${ }^{1}$, A.V.C. Silva ${ }^{2}$, \\ M.F. Arrigoni-Blank ${ }^{1}$, S.V.A. Carvalho ${ }^{1}$ and A.F. Blank ${ }^{1}$ \\ ${ }^{1}$ Laboratório de Recursos Genéticos Vegetais e Óleos Essenciais, \\ Departamento de Engenharia Agronômica, Universidade Federal de Sergipe, \\ São Cristóvão, SE, Brasil \\ ${ }^{2}$ Laboratório de Biotecnologia Molecular, Embrapa Tabuleiros Costeiros, \\ Aracaju, SE, Brasil \\ Corresponding author: A.F. Blank \\ E-mail: afblank@ufs.br
}

Genet. Mol. Res. 15 (4): gmr15049198

Received September 6, 2016

Accepted October 14, 2016

Published December 2, 2016

DOI http://dx.doi.org/10.4238/gmr15049198

Copyright (C) 2016 The Authors. This is an open-access article distributed under the terms of the Creative Commons Attribution ShareAlike (CC BY-SA) 4.0 License.

ABSTRACT. Myrcia lundiana Kiaersk. is a tree of the family Myrtaceae
found in tropical and subtropical areas of the southern hemisphere
that produces essential oil. The aim of this study was to characterize
the genetic diversity of $M$. lundiana plants from a native population
of Parque Nacional de Itabaiana, using inter-simple sequence repeat
molecular markers. Thirty-five primers were tested, 20 of which were
polymorphic, resulting in 135 polymorphic and informative bands.
Results of the cluster analysis, obtained using the unweighted pair
group method with arithmetic mean, grouped plants into three clusters:
Cluster I - MLU001, MLU002, MLU003, MLU004, MLU005,
MLU006, MLU018, MLU019, MLU020, MLU021, MLU022;

Genetics and Molecular Research 15 (4): gmr15049198 
MLU008, MLU011, MLU012, MLU014, MLU015, MLU017, MLU026, and MLU028; Cluster II - MLU007, MLU009, MLU010, MLU013, and MLU016; and Cluster III - MLU023, MLU024, MLU025, and MLU027. Jaccard similarity coefficients for pair-wise comparisons of plants ranged between 0.15 and 0.87. MLU014 and MLU015 presented low genetic diversity, with a similarity index of 0.87. Conversely, MLU007 and MLU019 presented high diversity, with a similarity index of 0.15 . According to the structure analysis, three distinct clusters were formed. Genetic diversity of $M$. lundiana plants was intermediate, and expansion of its genetic diversity is necessary. MLU026 and MLU028 are the most suitable for selection in breeding programs, since they clearly represent all of the diversity present in these plants. Moreover, these results provide important information on the existing genetic variability, highlighting the importance of Parque Nacional de Itabaiana for the conservation of this species.

Key words: Myrcia lundiana; Conservation; Genetic diversity; ISSR

\section{INTRODUCTION}

Myrcia lundiana Kiaersk, popularly known as "canela-de-tabuleiro", is a medicinal species of the family Myrtaceae, found in tropical and subtropical areas of the southern hemisphere (Govaerts et al., 2008). In folk medicine, species of the Myrcia genus have been used as astringents, diuretics, and for the treatment of diabetes mellitus, hypertension, gastric diseases, and diarrhea (Russo et al., 1990). Although little is known about the biological activity of $M$. lundiana, a recent study showed the antifungal potential of its essential oil against Lasiodiplodia theobromae (Alves et al., 2016).

The search for new molecules and bioactive substances from natural sources has highlighted the need for studies aiming to improve the conservation and maintenance of the genetic diversity of potentially useful plant species. The development of new roads, the construction of hydroelectric plants, and extractive activities, among other human activities, have greatly impacted the diversity of many species of the fauna and flora. Thus, it becomes necessary to manage genetic resources in order to preserve the maximum levels of genetic diversity within species. However, without information on the distribution of this variability between or within populations, important decisions regarding the management and conservation of these resources cannot be made (Lima et al., 2015). Knowledge on genetic variability and its organization in plant species, represent important steps for the exploitation, definition of genetic conservation strategies, and breeding programs (Setotaw et al., 2010). Owing to the importance of several medicinal species, either for popular use or for the pharmaceutical industry, several studies on genetic diversity in native populations have been carried out, such as those on Senna reticulata (Lima et al., 2015), Pilocarpus pennatifolius (Bandeira et al., 2010), Pothomorphe umbellata (Valle et al., 2013), and Annona crassiflora (Telles et al., 2003).

The study of genetic diversity in plant species is usually carried out using molecular markers. Among them, the inter-simple sequence repeats (ISSR) should be highlighted. These markers are dominant and permit the analysis of multiple loci in a single reaction owing to their

Genetics and Molecular Research 15 (4): gmr15049198 
abundance and dispersion within the genome. In addition, they exhibit high reproducibility, and can achieve results in a timely and cost-effective manner when compared with other markers (Rodrigues, 2010). ISSR markers are efficient at revealing genetic diversity among medicinal and agricultural plants, such as Varronia curassavica accessions and cassava landraces (Brito et al., 2016; Tiago et al., 2016).

Although limited information is available on the chemical diversity of the essential oil of M. lundiana in a native population from Sergipe (Alves et al., 2016), there are no reports in the literature regarding genetic studies of this species. Therefore, the objective of the present study was to analyze the genetic diversity of $M$. lundiana plants from a native population in the State of Sergipe using ISSR molecular markers.

\section{MATERIAL AND METHODS}

\section{Plant material}

Young leaves of $28 \mathrm{M}$. lundiana plants from of a native population of Parque Nacional de Itabaiana municipality of Areia Branca, State of Sergipe, Brazil, were collected (Table 1). After collection, leaves were wrapped in sterile gauze and stored on ice to prevent oxidation. The material was frozen at $-80^{\circ} \mathrm{C}$ until lyophilization in a LioTop (Liobras, São Carlos, SP, Brazil). After lyophilization, samples were stored in a desiccator containing silica gel until DNA extraction.

Table 1. Identification of 28 Myrcia lundiana plants from of a native population of Parque Nacional de Itabaiana, in the State of Sergipe, Brazil.

\begin{tabular}{|c|c|}
\hline Plant & Georeferenced information \\
\hline MLU001 & $11^{\circ} 44^{\prime} 17.9^{\prime \prime} \mathrm{S} ; 37^{\circ} 52^{\prime} 0.80^{\prime \prime} \mathrm{W}$ \\
\hline MLU002 & $10^{\circ} 44^{\prime} 57.0^{\prime \prime} \mathrm{S} ; 37^{\circ} 20^{\prime} 24.6^{\prime \prime} \mathrm{W}$ \\
\hline MLU003 & $10^{\circ} 44^{\prime} 58.0^{\prime \prime} \mathrm{S} ; 37^{\circ} 20^{\prime} 26.2^{\prime \prime} \mathrm{W}$ \\
\hline MLU004 & $10^{\circ} 44^{\prime} 58.0^{\prime \prime} \mathrm{S} ; 37^{\circ} 20^{\prime} 25.0^{\prime \prime} \mathrm{W}$ \\
\hline MLU005 & $10^{\circ} 44^{\prime} 57.7^{\prime \prime} \mathrm{S} ; 37^{\circ} 20^{\prime} 24.6^{\prime \prime} \mathrm{W}$ \\
\hline MLU006 & $10^{\circ} 44^{\prime} 57.5^{\prime \prime S} ; 37^{\circ} 20^{\prime} 26.0^{\prime \prime} \mathrm{W}$ \\
\hline MLU007 & $10^{\circ} 44^{\prime} 57.5^{\prime \prime} \mathrm{S} ; 37^{\circ} 20^{\prime} 26.1^{\prime \prime W}$ \\
\hline MLU008 & $10^{\circ} 44^{\prime} 56.2^{\prime \prime} \mathrm{S} ; 37^{\circ} 20^{\prime} 26.3^{\prime \prime} \mathrm{W}$ \\
\hline MLU009 & $10^{\circ} 44^{\prime} 58.1^{\prime \prime S} ; 37^{\circ} 20^{\prime} 26.1^{\prime \prime W}$ \\
\hline MLU010 & $10^{\circ} 45^{\prime} 00.0^{\prime \prime} \mathrm{S} ; 37^{\circ} 20^{\prime} 26.0^{\prime \prime} \mathrm{W}$ \\
\hline MLU011 & $10^{\circ} 44^{\prime} 57.7^{\prime \prime S} ; 37^{\circ} 20^{\prime} 24.3^{\prime \prime} \mathrm{W}$ \\
\hline MLU012 & $10^{\circ} 44^{\prime} 56.2^{\prime \prime} \mathrm{S} ; 37^{\circ} 20^{\prime} 26.5^{\prime \prime} \mathrm{W}$ \\
\hline MLU013 & $10^{\circ} 45^{\prime} 8.30^{\prime \prime} \mathrm{S} ; 37^{\circ} 20^{\prime} 27.5^{\prime \prime} \mathrm{W}$ \\
\hline MLU014 & $10^{\circ} 44^{\prime} 57.5^{\prime \prime} \mathrm{S} ; 37^{\circ} 20^{\prime} 26.5^{\prime \prime} \mathrm{W}$ \\
\hline MLU015 & $10^{\circ} 44^{\prime} 54.5^{\prime \prime} \mathrm{S} ; 37^{\circ} 20^{\prime} 27.9^{\prime \prime} \mathrm{W}$ \\
\hline MLU016 & $10^{\circ} 44^{\prime} 56.5^{\prime \prime} \mathrm{S} ; 37^{\circ} 20^{\prime} 24.0^{\prime \prime} \mathrm{W}$ \\
\hline MLU017 & $10^{\circ} 44^{\prime} 16.6^{\prime \prime} \mathrm{S} ; 37^{\circ} 52^{\prime} 00.5^{\prime \prime} \mathrm{W}$ \\
\hline MLU018 & $10^{\circ} 44^{\prime} 58.2^{\prime \prime} \mathrm{S} ; 37^{\circ} 20^{\prime} 26.1^{\prime \prime W}$ \\
\hline MLU019 & $10^{\circ} 44^{\prime} 56.8^{\prime \prime} \mathrm{S} ; 37^{\circ} 20^{\prime} 24.0^{\prime \prime} \mathrm{W}$ \\
\hline MLU020 & $10^{\circ} 44^{\prime} 57.5^{\prime \prime} \mathrm{S} ; 37^{\circ} 20^{\prime} 26.0^{\prime \prime} \mathrm{W}$ \\
\hline MLU021 & $10^{\circ} 44^{\prime} 57.6^{\prime \prime S} ; 37^{\circ} 20^{\prime} 26.1^{\prime \prime} \mathrm{W}$ \\
\hline MLU022 & $10^{\circ} 45^{\prime} 8.10^{\prime \prime} \mathrm{S} ; 37^{\circ} 20^{\prime} 17.5^{\prime \prime} \mathrm{W}$ \\
\hline MLU023 & $10^{\circ} 45^{\prime} 8.10^{\prime \prime} \mathrm{S} ; 37^{\circ} 20^{\prime} 18.5^{\prime \prime} \mathrm{W}$ \\
\hline MLU024 & $10^{\circ} 45^{\prime} 00.0^{\prime \prime} \mathrm{S} ; 37^{\circ} 20^{\prime} 26.0^{\prime \prime} \mathrm{W}$ \\
\hline MLU025 & $10^{\circ} 44^{\prime} 57.7^{\prime \prime S} ; 37^{\circ} 20^{\prime} 24.3^{\prime \prime} \mathrm{W}$ \\
\hline MLU026 & $10^{\circ} 45^{\prime} 00.0^{\prime \prime} \mathrm{S} ; 37^{\circ} 20^{\prime} 26.0^{\prime \prime} \mathrm{W}$ \\
\hline MLU027 & $10^{\circ} 44^{\prime} 57.7^{\prime \prime S} ; 37^{\circ} 20^{\prime} 24.3^{\prime \prime} \mathrm{W}$ \\
\hline MLU028 & $10^{\circ} 45^{\prime} 00.0^{\prime \prime} \mathrm{S} ; 37^{\circ} 20^{\prime} 26.0^{\prime \prime} \mathrm{W}$ \\
\hline
\end{tabular}

Genetics and Molecular Research 15 (4): gmr15049198 


\section{DNA extraction and PCR-ISSR amplification}

DNA extraction followed the procedures described by Doyle and Doyle (1990), modified as described by Alzate-Marin et al. (2005) to obtain DNA suitable for use in these experiments. DNA was diluted and subsequently standardized and quantified using the NanoDrop 2000c (Thermo Fisher Scientific, Wilmington, DE, USA). The ISSR primers used in this study were obtained from Eurofins MWG Operon (Operon Technologies, Louisville, KY, USA), IDT (Integrated DNA Technologies, Coralville, IA, USA), and Invitrogen (Thermo Fisher Scientific, Carlsbad, CA, USA). Thirty-five primers were tested for PCR amplification. PCR was carried out in a total volume of $20 \mu \mathrm{L}$, containing $1.0 \mu \mathrm{L}$ genomic DNA $(10 \mathrm{ng} / \mu \mathrm{L})$, $0.2 \mu \mathrm{L}$ recombinant Taq polymerase from Thermus aquaticus, expressed in Escherichia coli (Sigma-Aldrich, St. Louis, MO, USA) $(0.05 \mathrm{U} / \mu \mathrm{L})$, and $2 \mu \mathrm{L} 10 \mathrm{X}$ buffer $(100 \mathrm{mM}$ Tris-HCl, $\mathrm{pH} 8.3,500 \mathrm{mM} \mathrm{KCl}, 15 \mathrm{mM} \mathrm{MgCl}_{2}$, and $0.01 \%$ gelatin) (Sigma-Aldrich), $0.4 \mu \mathrm{L}$ dNTP (2.5 $\mathrm{mM}), 1.0 \mu \mathrm{L}$ primer $(25.0 \mathrm{pmol})$, and $15.4 \mu \mathrm{L}$ autoclaved ultrapure water.

PCR amplification was carried out in a ProFlex PCR thermocycler (Thermo Fisher Scientific, Applied Biosystems, Foster City, CA, USA) programmed with the following protocol: 5 min at $94^{\circ} \mathrm{C} ; 45$ cycles of $40 \mathrm{~s}$ at $94^{\circ} \mathrm{C} ; 30 \mathrm{~s}$ ranging from 50.4 to $53^{\circ} \mathrm{C}$, according to the annealing temperature used for each primer (Table 2); $1 \mathrm{~min}$ at $72^{\circ} \mathrm{C}$; and a final extension for $7 \mathrm{~min}$ at $72^{\circ} \mathrm{C}$. Amplification products were subjected to electrophoresis on $1.5 \%$ agarose gel, stained with ethidium bromide, visualized under ultraviolet light, and photodocumented. Molecular weights were estimated using a Ludwig DNA 1-kb scale for each primer.

\section{Data analysis}

The fragments of amplified DNA were analyzed for the presence (1) or absence (0) of equal-sized bands, and a binary matrix was constructed. Based on this binary matrix, the Jaccard similarity coefficient between each pair of individuals was calculated (Jaccard, 1908). Similarity coefficients were used to construct a dendrogram by the unweighted pair group method with arithmetic mean (UPGMA). These analyses were carried out using the NTSYSpc 2.0 software (Rohlf, 2001).

The Shannon index (I) and marker index (MI) were calculated using the software GENALEX 6.5 (Peakall and Smouse, 2012). STRUCTURE v.2.3.3 was used to analyze genetic structure using a Bayesian clustering method (Hubisz et al., 2009). The "admixture" model was used with correlated allele frequencies, and simulations were carried out with a burn-in of 100,000 generations and $\mathrm{K}$ values ranging from 2 to 6 clusters. The number of clusters (K) was determined using STRUCTURE HARVESTER (Earl and vonHoldt, 2012).

\section{RESULTS}

A high level of polymorphism was found in ISSR markers among the $28 \mathrm{M}$. lundiana plants from of a native population of Parque Nacional de Itabaiana, in the State of Sergipe, Brazil. Band locations can be visualized on the images generated by photodocumentation of the agarose gels (Figure 1). Of the 35 primers tested, 20 were polymorphic, since they presented good amplification patterns, with 144 amplified bands observed, of which 135 were polymorphic. This corresponds to $93.75 \%$ polymorphism, ranging from 3 (UBC826) to 17 (UBC815), and a mean number of 7.20 bands per primer (Table 2).

Genetics and Molecular Research 15 (4): gmr15049198 


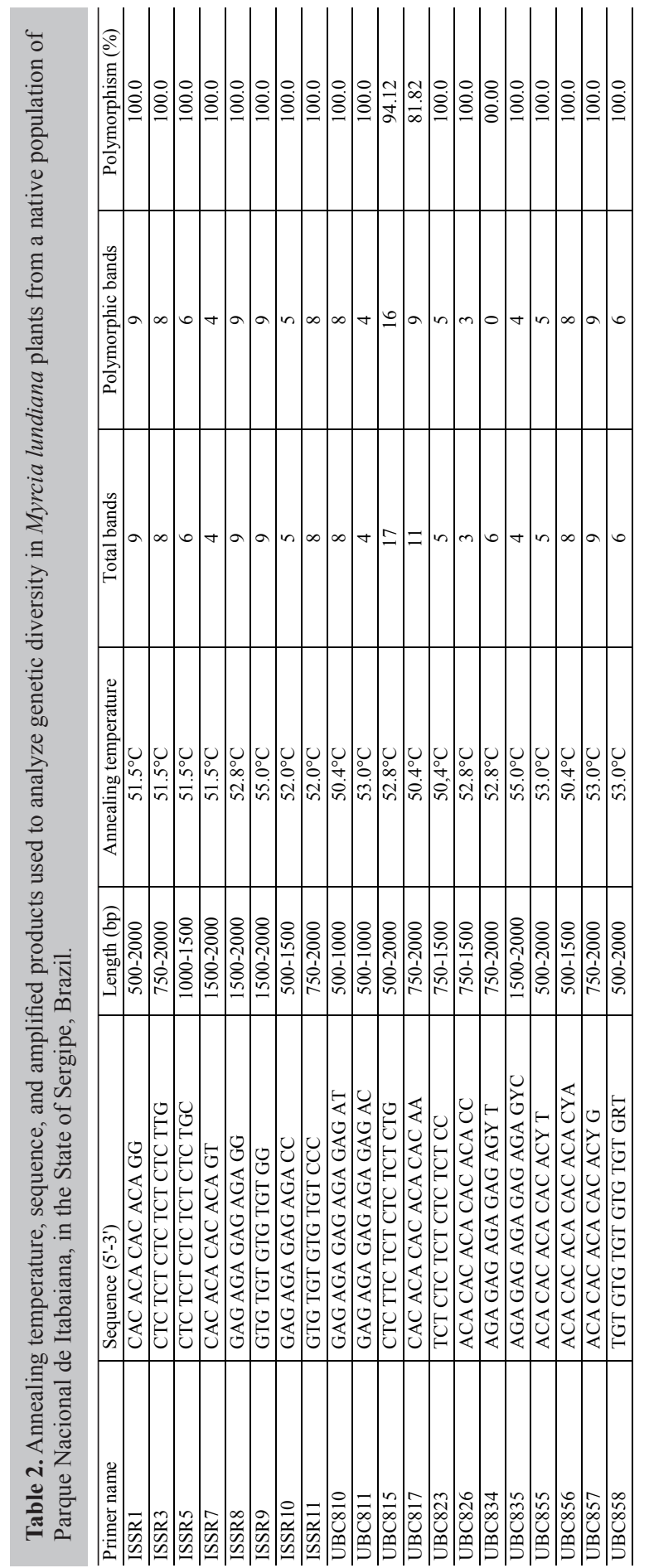

Genetics and Molecular Research 15 (4): gmr15049198 


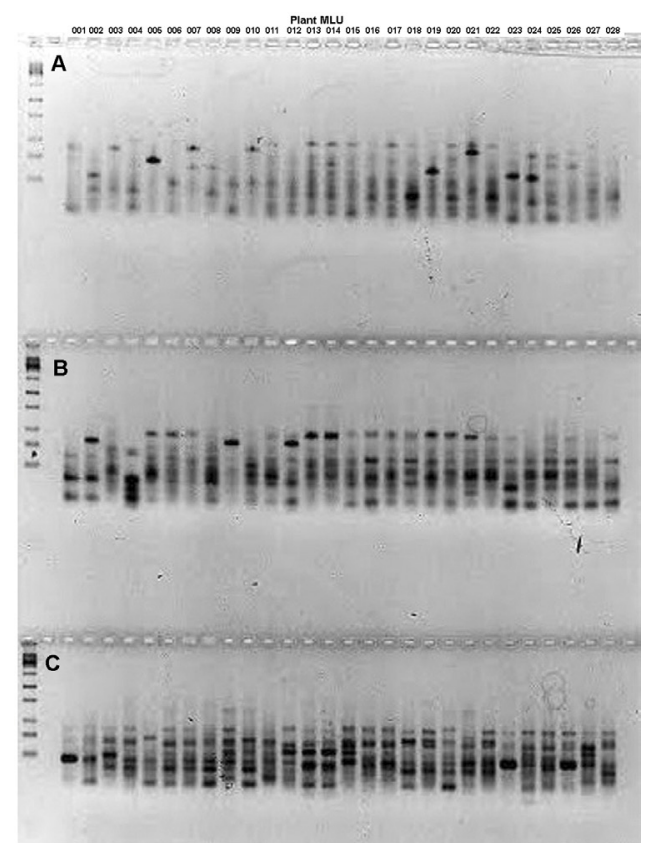

Figure 1. Agarose gels showing the electrophoretic profiles of the inter-simple sequence repeat markers amplified using the primers UBC856 (A), UBC823 (B), and UBC835 (C) in 28 Myrcia lundiana plants from of a native population of Parque Nacional de Itabaiana, in the State of Sergipe, Brazil.

The similarity coefficients used to calculate the genetic similarity between the $28 \mathrm{M}$. lundiana plants, as assessed by ISSR markers, ranged from 0.15 to 0.87 , with a mean value of 0.49 . Low genetic diversity was observed between MLU014 and MLU015, with a similarity index of 0.87 , representing the plants that were genetically most similar among all pairs assessed. Conversely, MLU007 and MLU019 exhibited the highest diversity, with a similarity index of 0.15 . There were 23 genotype combinations with intermediate genetic diversity $(0.60$ to 0.78 ), and other combinations with high genetic diversity, since their similarity values in the cluster were below 0.6 (0.24 to 0.59 ; Table 3$)$.

By means of clustering analysis, three clusters were formed according to the genetic distances between plants (Figure 2). Cluster I (67.86\% of plants) was formed by MLU001, MLU002, MLU003, MLU004, MLU005, MLU006, MLU018, MLU019, MLU020, MLU021, MLU022; MLU008, MLU011, MLU012, MLU014, MLU015, MLU017, MLU026, and MLU028, with Jaccard similarity indices between 0.4 and 0.87 ; Cluster II ( $17.86 \%$ of plants) presented similarity indices between 0.6 and 0.78 , and was formed by the plants MLU007, MLU009, MLU010, MLU013, and MLU016; and Cluster III (14.29\% of plants) showed similarity indices between 0.4 and 0.78 , and was formed by MLU023, MLU024, MLU025, and MLU027.

To determine the genetic patterns of population differentiation, Bayesian analysis was carried out by the Structure software and, according to the $\Delta \mathrm{K}$ method, the most likely number of clusters was $\mathrm{K}=3$ (Figure 3). Graphic visualization of the population structure permitted the identification of two plants, MLU026 and MLU028, which carried a mixture of the genetic material of the three clusters. 


\begin{tabular}{|c|c|c|c|c|c|c|c|c|c|c|c|c|c|c|c|c|c|c|c|c|c|}
\hline ष & $\sum 0$ & & & & & & & & & & & & & & & & & & & & \\
\hline త్రేี & $\frac{3}{2} \widehat{\delta}$ & & & & & & & & & & & & & & & & & & & & $\begin{array}{l}\vec{n} \\
0 \\
0\end{array}$ \\
\hline$\underset{0}{\tilde{Z}}$ & 远 & & & & & & & & & & & & & & & & & & & 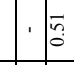 & 0 \\
\hline 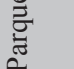 & קᄅ & & & & & & & & & & & & & & & & & & & 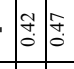 & $\begin{array}{l}0 \\
0 \\
0 \\
0 \\
\end{array}$ \\
\hline$\Xi$ & 层 & & & & & & & & & & & & & & & & & & & 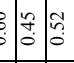 & fo \\
\hline $\begin{array}{l}4 \\
\text { t) } \\
\tilde{0}\end{array}$ & $\vec{\Sigma} \approx$ & & & & & & & & & & & & & & & & & & & 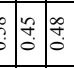 & fo \\
\hline 萦 & $\vec{Z} \approx$ & & & & & & & & & & & & & & & & & \begin{tabular}{ll|} 
\\
0 \\
0 \\
0
\end{tabular} & $b_{b}^{b}$ & $\begin{array}{lll}4 \\
\dot{b}\end{array}$ & $\begin{array}{l}8 \\
0 \\
0\end{array}$ \\
\hline $\begin{array}{l}\bar{\vdots} \\
0\end{array}$ & $\sum_{\bar{\Sigma}} \overline{\mathrm{s}}$ & & & & & & & & & & & & & & & & , & $\begin{array}{l}0 \\
\hat{n} \\
0 \\
0\end{array}$ & 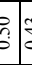 & 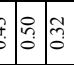 & 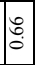 \\
\hline 丞 & उ & & & & & & & & & & & & & & & & $\begin{array}{lll}\infty \\
0 \\
0\end{array}$ & 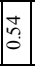 & 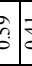 & $\therefore: \frac{0}{0}$ & $\begin{array}{l} \\
\\
0 \\
\end{array}$ \\
\hline$\frac{\pi}{4}$ & $\vec{\Sigma} \frac{\partial}{2}$ & & & & & & & & & & & & & & & $\begin{array}{l}2 \\
0 \\
0\end{array}$ & \begin{tabular}{ll}
$\circ$ \\
\hdashline
\end{tabular} & \begin{tabular}{|l|} 
\\
$\infty$ \\
0 \\
0 \\
\end{tabular} & $\stackrel{0}{0}$ & 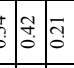 & $\begin{array}{l}n \\
0 \\
0 \\
0\end{array}$ \\
\hline ఏ్ & $\vec{\Sigma}^{\infty} \stackrel{\infty}{\circ}$ & & & & & & & & & & & & & & $\begin{array}{l}\overrightarrow{0} \\
0 \\
0\end{array}$ & $\div$ & $\begin{array}{ll}\infty & \vec{\infty} \\
& \vec{\infty} \\
\end{array}$ & $\begin{array}{l}n \\
\hat{n} \\
0 \\
0\end{array}$ & $\stackrel{0}{0}=$ & 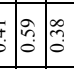 & : \\
\hline 泀 & $\vec{z}=$ & & & & & & & & & & & & & , $1 \begin{array}{l}\infty \\
n \\
0\end{array}$ & F. & $\mid$ & $\begin{array}{lll}0 \\
0\end{array}$ & $\begin{array}{l}\infty \\
\hat{0} \\
0 \\
0\end{array}$ & $\overbrace{0}^{\infty})$ & 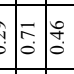 & $\overrightarrow{0}$ \\
\hline$\underset{\Xi}{\Xi}$ & 恿 & & & & & & & & & & & & & $\begin{array}{l}\text { J. } \\
\end{array}$ & $\frac{9}{0}$ & ড̇ & 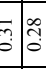 & \begin{tabular}{|l|} 
\\
\\
0 \\
0
\end{tabular} & 3 & $\begin{array}{lll}0 \\
\dot{f}\end{array}$ & ळ̂. \\
\hline 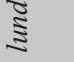 & $\sum_{2}^{2} \frac{n}{0}$ & & & & & & & & & & & & $\begin{array}{lll}\infty & 0 \\
0 & 0 & 0 \\
0 & 0 & 0\end{array}$ & 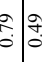 & 声 & t & $\begin{array}{lll}0 & 5 \\
6 & 0\end{array}$ & $\mid$ & $: \delta$ & 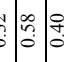 & $\begin{array}{l}1 \\
0 \\
0 \\
0 \\
0\end{array}$ \\
\hline 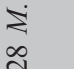 & $\frac{2}{2} \pm$ & & & & & & & & & & & & $\begin{array}{lll} & 0 \\
0 & 0 & 0 \\
0 & 0\end{array}$ & 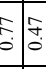 & f. & 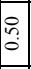 & $\begin{array}{c}6 \\
6\end{array}$ & 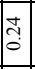 & $\vec{c}$ & $\hat{B} \theta$ & f. \\
\hline पै & 良 $\frac{m}{0}$ & & & & & & & & & & $1 \cdot \overrightarrow{\vec{n}}$ & & \begin{tabular}{lll}
0 \\
\hdashline \\
0
\end{tabular} & \begin{tabular}{lll} 
\\
\hdashline
\end{tabular} & ֻั & {$\left[\begin{array}{c}0 \\
0 \\
0 \\
0\end{array}\right.$} & $\begin{array}{lll}c & 0 \\
0 & 9 \\
0\end{array}$ & בิ & 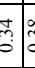 & 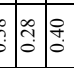 & 童 \\
\hline 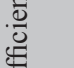 & $\frac{3}{\sum} \approx$ & & & & & & & & & & $\begin{array}{ll}\infty \\
0 \\
0 & \stackrel{N}{0} \\
\end{array}$ & & \begin{tabular}{|l|l}
$f$ & 5 \\
0 & 0 \\
0 & 0 \\
\end{tabular} & \begin{tabular}{l|l}
$:$ & \multirow{l}{\circ}{} \\
\end{tabular} & f & g. & 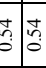 & 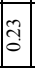 & ?ִ. & 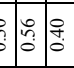 & 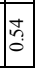 \\
\hline 8 & $\vec{z}=$ & & & & & & & & & $\begin{array}{lll}R & q \\
0 & 0\end{array}$ & 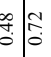 & & $\begin{array}{l}0 \\
\dot{a} \\
0\end{array}$ & \begin{tabular}{c}
8 \\
\hdashline \\
\hdashline
\end{tabular} & 离 & $\mid \begin{array}{l}n \\
n \\
0 \\
0 \\
0\end{array}$ & 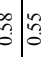 & $\mid$ & ç) & 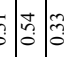 & in \\
\hline : & 골잉 & & & & & & & & . & $\hat{0}$ & : & & ర్d & 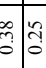 & 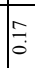 & 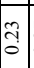 & $\begin{array}{ccc}\pi \\
\text { ç }\end{array}$ & के & 6 & 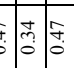 & ְִ \\
\hline है & ह & & & & & & & 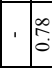 & 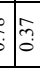 & के & 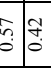 & & 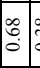 & 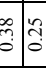 & $\stackrel{\infty}{0}$ & d. & 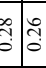 & \begin{tabular}{|c|} 
\\
$\tilde{m}$ \\
0 \\
\end{tabular} & 7 & 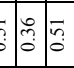 & \begin{tabular}{l}
$\infty$ \\
\multirow{2}{*}{} \\
0 \\
0
\end{tabular} \\
\hline 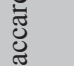 & 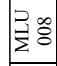 & & & & & & & \begin{tabular}{c|c}
$\infty$ & 0 \\
$\infty$ & 0 \\
0 & 0 \\
0
\end{tabular} & $: 0$ & $\sqrt[n]{0}$ & $\begin{array}{lll} \\
\end{array}$ & & $\tilde{b}$ & $\begin{array}{ll}6 & 5 \\
6\end{array}$ & $\tilde{2}$ & 活 & $\begin{array}{c}6 \\
0\end{array}$ & \begin{tabular}{|l|} 
\\
0 \\
0 \\
0
\end{tabular} & $\mathbb{J}$ & $\hat{b} \hat{b}=0$ & 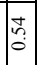 \\
\hline$\stackrel{\Xi}{\Xi} \cdot \dot{\bar{N}}$ & 方 & & & & & . & ? & \begin{tabular}{l|l}
0 & 0 \\
$:$ & 0 \\
0 & 0 \\
\end{tabular} & b. & 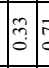 & 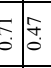 & & $:$ & \begin{tabular}{l|l}
\multirow{9}{q}{} & $\stackrel{0}{0}$ \\
0 & 0 \\
\end{tabular} & $\stackrel{2}{\circ}$ & 0 & \begin{tabular}{lll}
$\pi$ & \multicolumn{1}{c}{$c$} \\
\end{tabular} & \begin{tabular}{|l|l|} 
\\
0 \\
0 \\
\end{tabular} & $\tilde{c}$ & $\begin{array}{llll}0 & 0 \\
0 & 0 & 0 \\
0\end{array}$ & 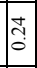 \\
\hline పี & $\frac{2}{2} \%$ & & & & & & 苾 & 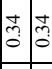 & & 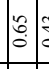 & $\begin{array}{l}f_{0} \\
\end{array}$ & & $\tilde{m}$ & \begin{tabular}{lll}
$\infty$ & 0 \\
\hdashline & 0 & 0 \\
0
\end{tabular} & in & & : & \begin{tabular}{|l|}
$\hat{2}$ \\
$\hat{0}$
\end{tabular} & $\stackrel{6}{6}$ & $\begin{array}{lll}0 \\
b \\
0\end{array}$ & $\begin{array}{l}1 \\
\text { ñ } \\
0\end{array}$ \\
\hline 胥 & $\sum_{2}$ & & & & & $\begin{array}{c}0 \\
\vdots \\
\vdots\end{array}$ & $\overrightarrow{0}$ & 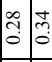 & $\overbrace{0}^{\circ}$ & â & $\begin{array}{llll}0 \\
0 \\
0\end{array}$ & & $\tilde{e}$ & \begin{tabular}{cc}
5 \\
\hdashline \\
\hdashline
\end{tabular} & : & 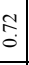 & $\overbrace{0}^{\circ}$ & $\mid \begin{array}{l}0 \\
0 \\
0 \\
0\end{array}$ & & 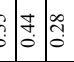 & की \\
\hline 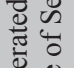 & 当 8 & & & & & $\begin{array}{l}\dot{B} \\
\dot{b}\end{array}$ & 0 & 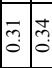 & की & $\tilde{0}$ & $\begin{array}{lll}\infty & 0 \\
0 & 0 & 0 \\
0 & 0 \\
\end{array}$ & & 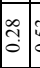 & $\hat{n}: \begin{array}{l}R \\
0 \\
0\end{array}$ & : & $\begin{array}{c}\mathbb{N} \\
\dot{0} \\
\end{array}$ & $\begin{array}{c}\infty \\
0 \\
0\end{array}$ & $\mid \begin{array}{l}0 \\
0 \\
0 \\
0\end{array}$ & $\dot{f}$ & 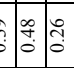 & ڤn \\
\hline 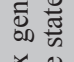 & $\stackrel{P}{\Sigma} \stackrel{8}{\Sigma}$ & & & & & 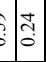 & & 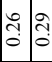 & $\vec{s}$ & 壳 & $\begin{array}{c}\text { ç: } \\
\text { ch }\end{array}$ & & $\tilde{m}_{0}^{0}$ & $\begin{array}{ll}\overrightarrow{0} \\
:\end{array}$ & $\begin{array}{l}\infty \\
n \\
0\end{array}$ & $\begin{array}{l}0 \\
: \\
0\end{array}$ & $\underbrace{}_{0}$ & $\mid$\begin{tabular}{l}
$\mid$ \\
\multirow{2}{*}{} \\
$\vdots$ \\
0 \\
0
\end{tabular} & n & $\begin{array}{l}n \\
b \\
0\end{array}$ & $\tilde{0}$ \\
\hline 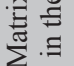 & 38 & & : & $\begin{array}{ll}\vec{\infty} \\
\dot{0} \\
\dot{0}\end{array}$ & & $\begin{array}{ll}0 \\
\vdots \\
0\end{array}$ & के & 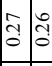 & : & $\begin{array}{l}I \\
\vdots \\
0 \\
0\end{array}$ & $\begin{array}{ll}9 & \pi \\
0 & 0 \\
0\end{array}$ & & $\stackrel{\infty}{\rightarrow}$ & 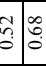 & $\ddot{0}$ & 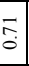 & 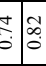 & 早 & : & $\begin{array}{lll}0 \\
\vdots\end{array}$ & S. \\
\hline ம் శే .ే & $\vec{z} \overline{8}$ & . & bे & 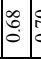 & & 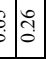 & $\overrightarrow{0}$ & \begin{tabular}{l|l}
$\substack{\pi \\
0}$ \\
\hdashline
\end{tabular} & 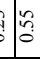 & $f$ & $\begin{array}{lll}0 \\
c \\
c\end{array}$ & ? & 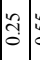 & \begin{tabular}{lll}
$\qquad$ \\
\hdashline
\end{tabular} & : & $\begin{array}{l}R \\
0 \\
0\end{array}$ & : & $\mid \begin{array}{l}0 \\
0 \\
0 \\
0\end{array}$ & $\begin{array}{l}0 \\
0 \\
0\end{array}$ & $\begin{array}{llll}0 & 0 \\
0 & 0 & 0 \\
0\end{array}$ & 㕝 \\
\hline 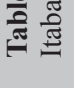 & 迹 & 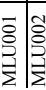 & 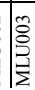 & 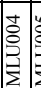 & & 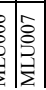 & & 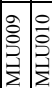 & 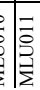 & 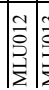 & 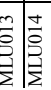 & & 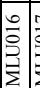 & 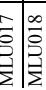 & 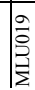 & & 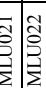 & 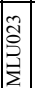 & & 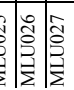 & 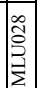 \\
\hline
\end{tabular}




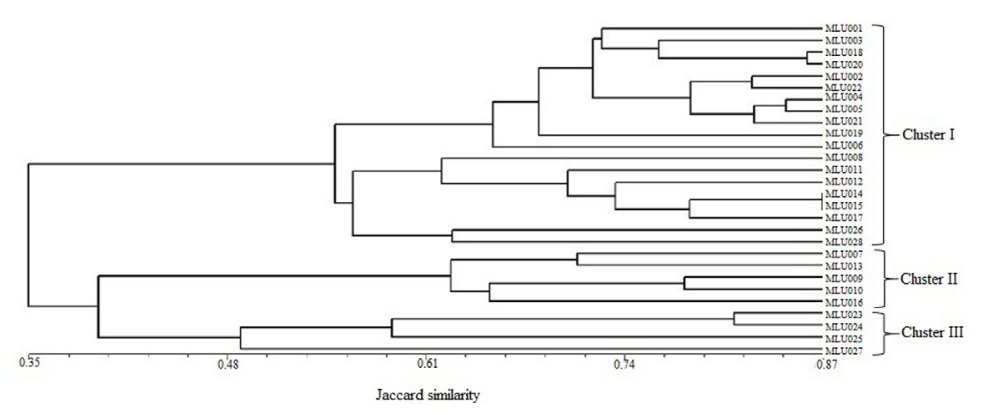

Figure 2. Dendrogram generated by the unweighted pair group method with arithmetic mean (UPGMA) analysis of Jaccard similarity indices for 28 Myrcia lundiana plants from of a native population of Parque Nacional de Itabaiana, in the State of Sergipe, Brazil.

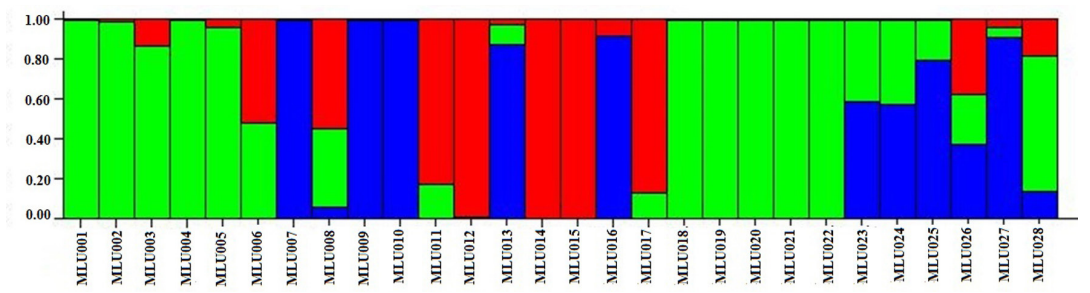

Figure 3. STRUCTURE results for 28 Myrcia lundiana plants from of a native population of Parque Nacional de Itabaiana, in the State of Sergipe, Brazil, with $\mathrm{K}=3$. Each vertical bar represents one accession, with color indicating cluster membership.

\section{DISCUSSION}

The native populations of $M$. lundiana were found to possess intermediate genetic diversity based on the values of the diversity parameters assessed, and by the division of the 28 plants into three clusters. The genetic diversity results obtained in this study were similar to those reported in a study on the chemical diversity of a population of M. lundiana by Alves et al. (2016), who detected high chemical diversity, and division into three chemical clusters. This indicates that studies using ISSR molecular markers should be extended in order to identify greater variability.

The Shannon index (I) presented a low mean value (0.46), which was below 0.5 . This index of genetic diversity may vary from 0 to 1 , with lower genetic diversity represented by values closest to zero (Silva et al., 2011). Heterozygosity, or the Nei index, which measures the genetic variability that estimates the variation between related individuals, was considered low, with a mean value of 0.30 .

The bar graph obtained by the Bayesian analysis using the Structure software showed the formation of three clusters defined by the colors blue, red, and green. MLU026 and MLU028 presented part of each cluster, which indicates that these plants are probably located within a transition point, and/or a region of intense exchange of genetic material. Such plants should be prioritized for preservation since they may be useful in future studies aimed at the genetic improvement of the species.

Little information is available on the history of the study area; however, vegetation corridors in the studied landscape are of great importance. The intermediate level of genetic 
diversity observed in the present study can be explained by the pollination type of $M$. lundiana (entomophily), by the dispersion of the diaspores (zoochory), by the proximity of the plants, and by the fact that they are interconnected by vegetation corridors.

Owing to the importance of many medicinal plant species for popular use, or even for use in the pharmaceutical industry, many studies on the genetic diversity of native populations have been carried out. In studies with $S$. reticulata and $P$. umbellata, high genetic diversity was observed within native populations (Valle et al., 2013; Lima et al., 2015). Bandeira et al. (2010) observed low genetic diversity between native populations of Jaborandi.

Few studies using ISSR markers have been reported in plants of the Myrtaceae family. When studying the spatial genetic structure in fine-scale of Myrcia splendens in environments with fragments and in their connections, Brandão et al. (2011) observed high levels of genetic diversity within populations. Oliveira et al. (2014), when studying the genetic distance between plants of the genus Psidium using ISSR markers, observed high variability between guava genotypes, which permits the crossing of superior genotypes with high divergence. Mani et al. (2011), when assessing the genetic distances in different species of Psidium spp, obtained a total of 234 polymorphic bands in 31 ISSR markers. Silva et al. (2011) assessed the variability of plants of the Manihot genus, and obtained a total of 154 polymorphic bands using 20 primers.

The use of modern techniques, such as those involving molecular markers, is extremely important in breeding programs. This is owing to the accuracy of the information present in the genome, since it is not affected by the environment.

According to Celestino et al. (2015), molecular tools are crucial for the characterization of genetic diversity in aromatic and medicinal plants, i.e., although the agronomic and chemical characteristics distinguish the genotypes of the clusters, the composition of essential oils can be altered by environmental factors.

Genetic diversity of $M$. lundiana plants from a native population of Parque Nacional de Itabaiana, in the State of Sergipe, Brazil, is intermediate. Plants MLU026 and MLU028 were the most suitable for selection in breeding programs of this species, since they carry all of the genetic diversity present in these plants. The results found in this study are important for the conservation of this species, and highlight the need to increase the number of molecular markers, as well as the number of samples for the study of genetic diversity in Parque Nacional de Itabaiana.

\section{Conflicts of interest}

The authors declare no conflict of interest.

\section{ACKNOWLEDGMENTS}

The authors thank CNPq, FAPITEC/SE, CAPES, and FINEP for their financial support of this study.

\section{REFERENCES}

Alves MF, Nizio DAC, Sampaio TS, do Nascimento AF, Jnr., et al. (2016). Myrcia lundiana Kiaersk native populations have different essential oil composition and antifungal activity against Lasiodiplodia theobromae. Ind. Crops Prod. 85: 266-273. http://dx.doi.org/10.1016/j.indcrop.2016.03.039

Genetics and Molecular Research 15 (4): gmr15049198 
Alzate-Marin AL, Guidugli MC, Soriani HH and Mestriner MA (2005). Otimização de um método econômico e rápido de extração de DNA para quatro espécies de árvores tropicais. In: Anais do $51^{\circ}$ Congresso Brasileiro de Genética, Águas de Lindóia.

Bandeira AJ, Deimling LI and Georg-Kraemer JE (2010). Variabilidade genética do jaborandi (Pilocarpus pennatifolius Lemaire; Rutaceae) em populações naturais da região noroeste do Rio Grande do Sul. Rev. Inic. Cient. ULBRA 8: 43-51.

Brandão MM, Vieria FA and Carvalho D (2011). Estrutura genética em microescala espacial de Myrcia splendens (Myrtaceae). Rev. Arvore 35: 957-964. http://dx.doi.org/10.1590/S0100-67622011000600001

Brito FA, Nizio DAC, Silva AV, Diniz LE, et al. (2016). Genetic diversity analysis of Varronia curassavica Jacq. accessions using ISSR markers. Genet. Mol. Res. 15: http://dx.doi.org/10.4238/gmr.15038681.

Celestino RS, Zucchi MI, Pinheiro JB, Campos JB, et al. (2015). Molecular and chemical characterization of vetiver, Chrysopogon zizanioides (L.) Roberty, germplasm. Genet. Mol. Res. 14: 9452-9468. http://dx.doi.org/10.4238/2015. August.14.9

Doyle JJ and Doyle JL (1990). Isolation of plant DNA from fresh tissue. Focus 12: 13-15.

Earl DA and vonHoldt BM (2012). STRUCTURE HARVESTER: a website and program for visualizing STRUCTURE output and implementing the Evanno method. Conserv. Genet. Resour. 4: 359-361. http://dx.doi.org/10.1007/ $\underline{\text { s12686-011-9548-7 }}$

Govaerts R, Sobral M, Ashton P, Barrie F, et al. (2008). World checklist of Myrtaceae. Royal Botanic Gardens, Kew. $455 \mathrm{p}$.

Hubisz MJ, Falush D, Stephens M and Pritchard JK (2009). Inferring weak population structure with the assistance of sample group information. Mol. Ecol. Resour. 9: 1322-1332.http://dx.doi.org/10.1111/j.1755-0998.2009.02591.x

Jaccard P (1908). Nouvelles recherches sur la distribution florale. Bull. Soc. Vaud. Sci. Nat. 44: 223-270.

Lima RA, Lopes MTG, Bentes JLS, Valente MSF, et al. (2015). Diversidade e estrutura genética de Senna reticulata. Floresta 45: 507-514. http://dx.doi.org/10.5380/rf.v45i3.38079

Mani A, Mishra R and Thomas G (2011). Elucidation of diversity among Psidium species using morphological and SPAR methods. J. Phytol. 3: 53-61.

Oliveira NNS, Viana AP, Quintal SSR, Paiva CL, et al. (2014). Análise de distância genética entre plantass do gênero Psidium via marcadores ISSR. Rev. Bras. Frutic. 36: 917-923. http://dx.doi.org/10.1590/0100-2945-413/13

Peakall R and Smouse PE (2012). GenAlEx 6.5: genetic analysis in Excel. Population genetic software for teaching and research-an update. Bioinformatics 28: 2537-2539. http://dx.doi.org/10.1093/bioinformatics/bts460

Rodrigues JF (2010). Delimitação de espécies e diversidade genética no complexo Cattleya coccinea Lindl. e C. mantiqueirae (Flowie) Van der Berg (Orchidaceae) baseada em marcadores moleculares ISSR. Master's thesis. ESALQ/USP, Piracicaba.

Rohlf FJ (2001). NTSYSpc: numerical taxonomy system, Version 2.0. Exeter Publishing, Setauket.

Russo EM, Reichelt AA, De-Sá JR, Furlanetto RP, et al. (1990). Clinical trial of Myrcia uniflora and Bauhinia forficata leaf extracts in normal and diabetic patients. Braz. J. Med. Biol. Res. 23: 11-20.

Setotaw TA, Dias LDS and Missio RF (2010). Genetic divergence among barley accessions from Ethiopia. Crop Breed. Appl. Biotechnol. 10: 116-123. http://dx.doi.org/10.12702/1984-7033.v10n02a03

Silva KVP, Alves AAC, Martins MIG, Melo CAF, et al. (2011). Variabilidade genética entre plantas do gênero Manihot por meio de marcadores moleculares ISSR. Pesqui. Agropecu. Bras. 46: 1082-1088. http://dx.doi.org/10.1590/ $\underline{\text { S0100-204X2011000900016 }}$

Telles MPC, Valva FD, Bandeira LF and Coelho AG (2003). Caracterização genética de populações naturais de araticunzeiro (Annona crassiflora Mart. - Annonaceae) no Estado de Goiás. Rev. Brasil. Bot. 26: 123-129.

Tiago AV, Rossi AAB, Tiago PV, Carpejani AA, et al. (2016). Genetic diversity in cassava landraces grown on farms in Alta Floresta-MT, Brazil. Genet. Mol. Res. 15: http://dx.doi.org/10.4238/gmr.15038615.

Valle JS, Fonseca BKD, Nakamura SS, Linde GA, et al. (2013). Diversidade genética de populações naturais de pariparoba [Pothomorphe umbellata (L.) Miq.] por RAPD. Rev. Bras. Plantas Med. 15: 47-53. http://dx.doi.org/10.1590/S1516$\underline{05722013000100006}$

Genetics and Molecular Research 15 (4): gmr15049198 